\title{
Synergistic modification of the tribological properties of polytetrafluoroethylene with polyimide and boron nitride
}

\author{
Chaojie XIE, Kejian WANG* \\ College of Mechanical and Electrical Engineering, Beijing University of Chemical Technology, Beijing 100029, China \\ Received: 29 April 2020 / Revised: 01 July 2020 / Accepted: 11 July 2020 \\ (C) The author(s) 2020 .
}

\begin{abstract}
Polytetrafluoroethylene (PTFE) blended with polyimide (PI) and filled with boron nitride (BN) is prepared through cold pressing and sintering for composites with remarkable wear resistance and reduced coefficient of friction (COF). The characterizations show that BN and PI at different levels, improve the hardness, dynamic thermo-mechanical modulus, thermal conductivity, and tribological properties of PTFE. PI boosts the dispersion and bonding of BN in PTFE. In dry sliding friction of a block-on-ring tribometer, the wear rate and COF of 10:10:80 BN/PI/PTFE reduce to almost 1/300 and 80\% of those of pure PTFE, respectively, as the wear mechanism transition from being adhesive to partially abrasive. This occurs only when the additives BN and PI induce a synergistic effect, that is, at concentrations that are not higher than $c a .10 \mathrm{wt} \%$ and $15 \mathrm{wt} \%$, respectively. The obvious agglomeration at high percentages of added PI and severe conditions (400 N and $400 \mathrm{rpm}$ ) induce strong adhesive failure. The variations in the tensile properties, hardness, crystallization, and microstructure of the composites correspond to different effects. The multiple parameters of the plots of wear and friction are transformed into their contour curves. The mechanism transition maps aid in understanding the influence of various test conditions and composite compositions on the contact surfaces in the space-time framework of wear.
\end{abstract}

Keywords: wear mechanism; coefficient of friction (COF); transition map; polytetrafluoroethylene (PTFE); polyimide (PI); boron nitride(BN)

\section{Introduction}

In chemical industries [1], hydraulic engineering [2], and aerospace mechanisms [3], some dynamic mechanical transmissions require close sealing at the contacting interfaces between relative moving components to prevent liquid leakage. A long-standing, steady, and low abrasion sealing is necessary for the reliable operation of equipment throughout their service life. More importantly, many operational conditions involve corrosive environments, high temperatures, and long durations of continuous operation [1-3]. Thus, sealing rings must be fabricated from materials which are corrosion-resistant, abrasion-resistant, thermostable, and with a low coefficient of friction (COF). These multiple and steep requirements can only be satisfied by employing composites. Therefore, the development of composite sealing materials with superior performances is significant to assure the reliability and improve the efficiency and increase the working life of machines and moving parts.

Polytetrafluoroethylene (PTFE) is widely used in mechanical seal structures due to its chemical stability, high self-lubrication, and heat resistance, as well as its mechanical properties [4]. However, in recent years, seals are required to operate under higher speeds, heavier pressures, and higher temperatures in more corrosive environments. Therefore, various

* Corresponding author: Kejian WANG, E-mail: wangkj@mail.buct.edu.cn 
modifications of PTFE have been conducted to improve its properties to endure such severe conditions [5]. Certain properties of the adopted modifiers must be superior to those of PTFE [6]; or at least, the modifier should cause synergistic effects with PTFE [7]. Thus, some modifier candidates include engineering resins and inorganic fillers, which are capable of working at high temperatures to improve the energy dissipation [8] and thermo-deformation [9] of PTFE. Good tribological properties imply both low abrasion rates and low COFs [10]. Abrasion-resistant additives should be at least partially compatible with the PTFE matrix for uniform blending to ensure good cohesive bonding between the materials and reduce the material loss during operation [11, 12]. Some possible polymer additives include thermoset resins [13] and thermoplastics, such as polyphenylene sulfide [14, 15], polyether-ether ketone [16, 17], fluorinated copolymer [17], multiple component blends [18], and polyimide (PI) [19]. The modifications using these polymers can result in the production of materials that meet the requirements of high load ultrasonic motors [19-20]. Among them, PI is the most commonly used, for its excellent mechanical properties and wear-resistant strength. However, its relatively higher COF (ca. 0.3-0.4) than that of PTFE discounts its advantageous properties [19].

Materials with a lower coefficient of rolling friction than coefficient of sliding friction experience less wear [21]. In composites, the filler particles, regardless of their bonding in the matrix, can cause a similar action when the abrading surface of the counter body slides relatively to them. [21] At present, wearresistant PTFEs are commonly filled with particles such as $\mathrm{SiO}_{2}$ [22], alumina nanoparticles [23], molybdenum [24], carbon nanotubes [25], graphite [26], boron nitride [27], hybrids of ceramic, metal, and inorganic powders/particles [28], and even with fabric materials to effectively enhance its anti-friction tribological properties. Ref. [31] showed that boron nitride (BN) can improve the thermal, dielectric, and mechanical properties of PTFE [29] and PI [30], aside from their tribological properties.

The synergistic effects between organic polymers and inorganic fillers are also expected to significantly improve the properties of PTFE-based composites [32]. Such significant improvements were obtained by blending PTFE with PI and filling the composite with copper nanoparticles [33], polyhedral oligomeric silsesquioxane [34], and graphite [35] among others. So far, there have been few reports on the friction and wear characteristics of PTFE modified by PI and BN, whose effects are presented in this study.

Complex composites are expected to endure mechanical deformation under industrial conditions. For instance, a suitable PTFE composite sealing ring must exhibit excellent tensile strength and elongation at break (more than 150\%) when working against rubber in aviation applications. Thus, in ameliorating tribological behaviors, it must be considered whether the mechanical properties of the composite material meet the requirements of its technical applications. However, many studies consider only some properties of the composite and neglect others.

Several works have described the differential friction and wear mechanisms of materials and their relationship with the coupling material [36], surface asperity [37], running parameters [38-40], and relative motion of counterparts [41], as well as their performance in dry or aqueous environments [42]. Recently, the difference in the behaviors of these materials has been related to the variations in multiscale structures [42, 43], thermal states [44, 45], mechanical properties [45], heat transfer [46], and rates of wear and friction [47]. Therefore, it is widely accepted that direct tribological tests are necessary for tracing these causes in both theoretical and experimental studies to obtain unified wear frameworks. The effects of filler content, shape, interface, and size on the properties of composites are widely studied [48]. The present work is limited to determining the influence of blending a second polymer into the primary matrix and the use of $\mathrm{BN}$ as a filler in a PTFE-based composite.

In this study, a modified PTFE matrix composite was prepared with BN and PI at different proportions through the cold pressing and sintering processes. The composites were characterized using tribometry, mechanics, dynamic thermodynamics, and micromor- 
phology. Their friction and wear characteristics under different friction loads and rotation speeds were determined to provide a technical and quantitative basis for their application in industrial sealing materials with superior performance.

\section{Experimental}

\subsection{Materials}

Polyflon PTFE M-18F, with a size of $25 \mu \mathrm{m}$ and bulk density ca. $2.19 \mathrm{~g} / \mathrm{cm}^{3}$, was obtained from Daikin Industries Co., Ltd. BN, with a molecular weight of $24.84 \mathrm{~g} / \mathrm{mol}$ and density ca. $2.29 \mathrm{~g} / \mathrm{cm}^{3}$, was sourced from Tianyuan Chemical. Translucent amber YS-20 PI was obtained from Shanghai Synthetic Resin Factory.

\subsection{Specimen preparations}

PTFE powders were placed in an oven at $5{ }^{\circ} \mathrm{C}$ for $6 \mathrm{~h}$. Thereafter, PTFE, PI, and BN powders were weighed according to the proportions shown in Table 1 and were mixed for $4 \mathrm{~min}$ in a DAC 400 VAC high-speed mixer produced by FlackTek Inc. The mixtures had a uniform distribution after mixing twice. Subsequently, they were kept in an oven at $25{ }^{\circ} \mathrm{C}$ for $24 \mathrm{~h}$.

Each treated mixture was then transferred into a mold and compressed densely between flat platens using a YM-C100T hydraulic press manufactured by Wuxi Yangming Rubber Machinery Co., Ltd. The pressure was maintained at $50 \mathrm{MPa}$ for $30 \mathrm{~min}$.

Pre-compacted square planks with an area of $80 \mathrm{~mm} \times 80 \mathrm{~mm}$ and thickness of $8 \mathrm{~mm}$ of pure PTFE or the powder mixtures were taken out of the mold

Table 1 Specimen formulations.

\begin{tabular}{cccc}
\hline \multirow{2}{*}{ Sample No. } & \multicolumn{3}{c}{ Composition $(\mathrm{wt} \%)$} \\
\cline { 2 - 4 } & PTFE & BN & PI \\
\hline 1 & 100 & 0 & 0 \\
2 & 90 & 10 & 0 \\
3 & 85 & 10 & 5 \\
4 & 83 & 10 & 7 \\
5 & 80 & 10 & 10 \\
6 & 75 & 10 & 15 \\
7 & 70 & 10 & 20 \\
\hline
\end{tabular}

and cooled in the air at $25{ }^{\circ} \mathrm{C}$ for $24 \mathrm{~h}$. Thereafter, the planks were placed on a flat steel plate and moved into a GF14Q sintering furnace fabricated by Nanjing Boyuntong Instrument Technology Co., Ltd. The sintering was carried out under atmospheric pressure, and the planks were heated by the radiation from the electric wire fixed inside the stove wall together with the convection of high-temperature air in the closed furnace. The heating schedule for the sintering process is shown in Fig. 1. The full sintering cycle without warp lasted for $18 \mathrm{~h}$, including the rapid heating at $60{ }^{\circ} \mathrm{C} / \mathrm{h}$ from room temperature to $315{ }^{\circ} \mathrm{C}$, slow heating at $30{ }^{\circ} \mathrm{C} / \mathrm{h}$ to $360{ }^{\circ} \mathrm{C}, 4.5 \mathrm{~h}$ at a constant high temperature of $360{ }^{\circ} \mathrm{C}$, slow cooling at $30{ }^{\circ} \mathrm{C} / \mathrm{h}$ to $300{ }^{\circ} \mathrm{C}$, and rapid cooling at $50{ }^{\circ} \mathrm{C} / \mathrm{h}$ to $40{ }^{\circ} \mathrm{C}$. Finally, the sintered planks were taken out of the furnace and allowed to relax in air.

The fully sintered and relaxed planks were then cut into different test specimens with sizes according to certain standards using a CNC milling machine.

\subsection{Tests}

\subsubsection{Mechanical test}

V-type dumbbell-shaped specimens according to ASTM-D638-2003 were drawn using an XWWW$10 \mathrm{KN}$ universal machine from Beijing Jinshengxin Testing Instrument Co., Ltd. Stress-strain curves were recorded to determine the tensile properties of the composites. The reported values are the average results from five specimens.

\subsubsection{Friction and wear test}

This test was performed in a dry sliding condition using MRH-1 block-on-ring friction and wear tester

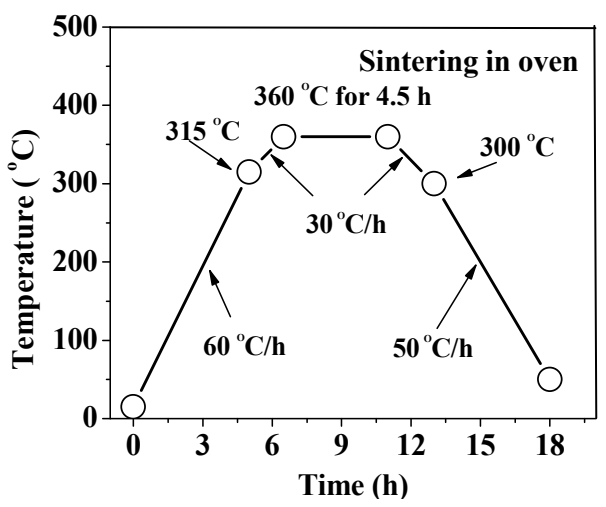

Fig. 1 Heating schedule for sintering. 
by Jinan Yihua Tribometer Technology Co., Ltd. The counterpart in friction was a cylindrical rotator made of 45 steel with an outside diameter of $40 \mathrm{~mm}$ and surface roughness of $0.25-0.35 \mu \mathrm{m}$, according to the standard GB/T 3960-2016 (corresponds to ASTM D2714-94). The ring rotated, wearing the top horizontal flat specimen by a certain loaded weight. The surface temperature of the specimen was monitored using an infrared thermometer.

The composite specimens with dimensions of $30 \mathrm{~mm} \times 7 \mathrm{~mm} \times 6 \mathrm{~mm}$ were ultrasonically cleaned with an acetone solution and subsequently dried by a draught fan. The specimens were weighed before and after the tribological experiment using an electronic analytical balance with an accuracy of 0.1 mg. The difference between the initial weight and the final weight was taken as the weight loss, representing the bulk wear consumption of the specimen. The specific wear rate $(\omega)$ was calculated using the expression $\omega=V / P L$, where $V$ is the volume of the consumed material during wearing in $\mathrm{mm}^{3}, P$ is the test load in $\mathrm{N}$, and $L$ is the product of the rotation speed and the outer circumference of the friction ring.

\subsubsection{Hardness test}

The Shore hardness of the specimens was determined using a hardness tester (XHS-D) in the atmosphere, following ISO 7619.

\subsubsection{Dynamic mechanical thermal analysis (DMTA)}

Dynamic mechanical tests were carried out in a three-point bending mode using a TA Instrument DMTA Q800 equipped with a liquid nitrogen apparatus. The frequency used was $10 \mathrm{~Hz}$, and the displacement amplitude was $2 \times 10^{-5} \mathrm{~m}$. The heating rate was $3.0{ }^{\circ} \mathrm{C} / \mathrm{min}$, and the experiments were carried out from 20 to $350{ }^{\circ} \mathrm{C}$. The specimen dimensions were $50 \mathrm{~mm} \times 20 \mathrm{~mm} \times 4 \mathrm{~mm}$.

\subsubsection{Differential scanning calorimetry (DSC)}

Thermal analysis was performed using a TA Instrument DSC Q2000 in a nitrogen atmosphere. The instrument was calibrated with standard indium. The samples (about 5-10.0 mg in mass) from the sintered specimens were first heated up to $350{ }^{\circ} \mathrm{C}$ at the heating rate of $20{ }^{\circ} \mathrm{C} / \mathrm{min}$, held at this temperature for $3 \mathrm{~min}$ to eliminate their thermal history, quenched to $30{ }^{\circ} \mathrm{C}$, and then reheated to $350{ }^{\circ} \mathrm{C}$ at $10{ }^{\circ} \mathrm{C} / \mathrm{min}$. The thermograms were recorded.

\subsubsection{Thermal conductivity measurement}

Thermal conductivity $(\lambda)$ was measured using the two-plate method through a TC3000 tester by Xi'an Electronic Technology Co., Ltd. The dimensions of the specimen were $30 \mathrm{~mm} \times 30 \mathrm{~mm} \times 4 \mathrm{~mm}$. The collection voltage was $1.5 \mathrm{~V}$, and the testing time was $5 \mathrm{~s}$.

The above measurements were repeated at least thrice, and the average $\lambda$ was taken to ensure the correctness and representativeness of the measurement.

\subsubsection{Microstructural observation}

The friction of the specimen surface was observed under an S6IF electron-optical microscope produced by the Sixth Shanghai Optical Instrument Factory.

\section{Results and discussion}

\subsection{Tensile properties of composites}

The effects of the modifications of adding BN and blending with PI on the tensile strength and the elongation at break of the PTFE composites are shown in Fig. 2. The thermoplastic PTFE exhibits a breakage-resistance higher than that of PI. The tensile strength of pure PTFE can reach $63 \mathrm{MPa}$, and its elongation at break is $380 \%$. After $10 \mathrm{wt} \%$ of the BN particles are filled into PTFE, the tensile properties of the resultant composite decrease by

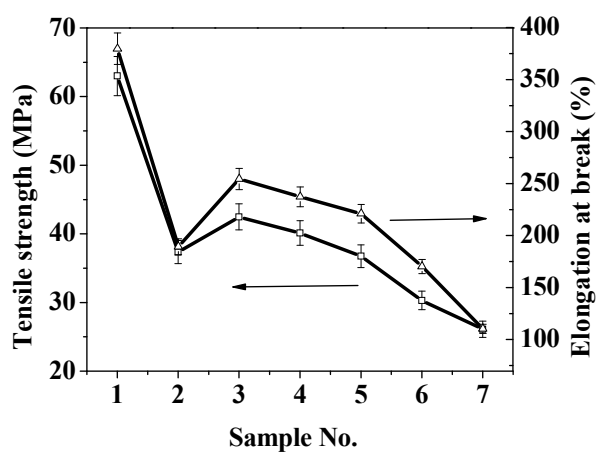

Fig. 2 Tensile properties of PTFE and its composites with $\mathrm{BN}$ and PI. 
almost half of that of pure PTFE. The rigid inorganic BN particles are successfully dispersed and embedded in the matrix through mechanical mixing. However, the particles and their agglomerates damage the continuity of PTFE. Significant local defects are also formed, introducing stress concentrations within the composite. As a result, the tensile strength and elongation at break of the 10:90 BN/PTFE composite are greatly reduced to $37.4 \mathrm{MPa}$ and $189 \%$, respectively.

As expected, the impairment caused by the addition of a fixed content $(10 \mathrm{wt} \%)$ of BN fillers is ameliorated by blending PTFE with the polar polymer PI. The incorporation of $5 \mathrm{wt} \%$ PI significantly improves the tensile strength and elongation at the break of the composite. At low concentrations, PI distributes well in PTFE and bonds with the dispersed BN, increasing the interfacial area between the PI and PTFE domains. The improved uniform structure of the three phases is responsible for the increased plasticity of the composite.

However, at higher concentrations of blended PI, the distribution of the different phases becomes deteriorated, and more local defects occur, reducing the properties of the composite. This indicates that the PI content should be kept below $10 \mathrm{wt} \%$ to obtain a BN/PI/PTFE composite with better tensile properties than the 10:90 BN/PTFE composite. One of the industrial customers requires the elongation at the break of the composite sealing ring to be more than $150 \%$. The results show that the 10:15:75 $\mathrm{BN} / \mathrm{PI} / \mathrm{PTFE}$ composite satisfies this requirement, with tensile strength and elongation at break of $30.3 \mathrm{MPa}$ and $171 \%$, respectively. However, when the PI content is increased to $20 \%$, the tensile strength and elongation at break of the composite fall to $26 \mathrm{MPa}$ and $110 \%$, respectively, which are about $1 / 3$ of those of PTFE. This is because interfacial debonding and defect failure occurs when composites with more aggregations of PI domains are subjected to an external tensile force.

\subsection{Hardness of composites}

$\mathrm{BN}$ is more rigid than molecular PTFE, rendering the 10:90 BN/PTFE composite (56 HD) to be harder than pure PTFE (52 HD). Furthermore, the rigidity of the PI molecule is greater than that of the PTFE molecule, making the BN/PI/PTFE composites to be harder than the PI. In terms of structure, the BN particles reinforce the interlocked boundary of the PI/PTFE blend. The combination of these two effects is reflected by the substantial increment in hardness from PTFE to BN/PTFE and from BN/ PTFE to BN/ PI/PTFE. The rise in hardness is relatively gradual due to the addition of PI in the three-component composite. When the PI content is increased to $20 \%$, the hardness of the composite can reach $65 \mathrm{HD}$. All of these are illustrated in Fig. 3. Indeed, the modulus and hardness of PI are higher than those of pure PTFE. Composites filled with hard BN bear larger external compressive loads, which is favorable to having wear-resistance in friction.

\subsection{Thermomechanical properties of composites}

Friction induces the deformation of a part of the composite by transforming the mechanical load into heat. Accumulated heat softens the material, which in turn triggers a greater degree of deformation and wear loss. Thus, appropriate modifications should be carried out to ensure the enhancement in the dynamic thermo-mechanical properties of the composites, which are reported here.

Two prominent peaks are on the curves of the loss tangent of PTFE and its composites in DMTA, as shown in Fig. 4(a). The peak at $c a .138{ }^{\circ} \mathrm{C}$ appears in the curves for PTFE and BN/PTFE, whereas the lowtemperature peak for the PTFE component slightly shifts to a higher temperature, up to $c a .140{ }^{\circ} \mathrm{C}$, for 10:20:70 BN/PI/PTFE. Another high-temperature peak is observed at $c a .286{ }^{\circ} \mathrm{C}$, corresponding to the PI component upon the addition of PI in BN/PTFE.

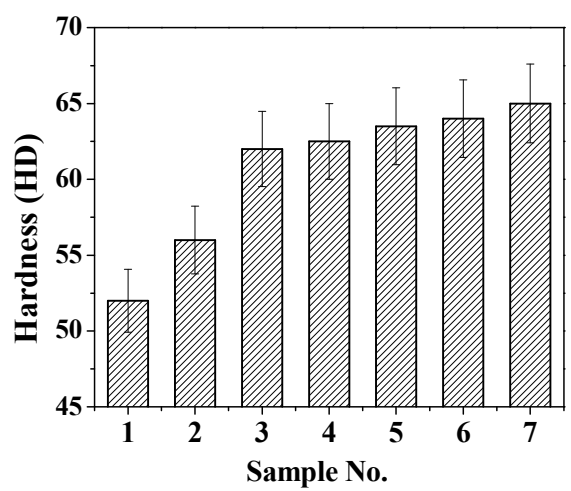

Fig. 3 Hardness of PTFE and its composites with BN and PI. 

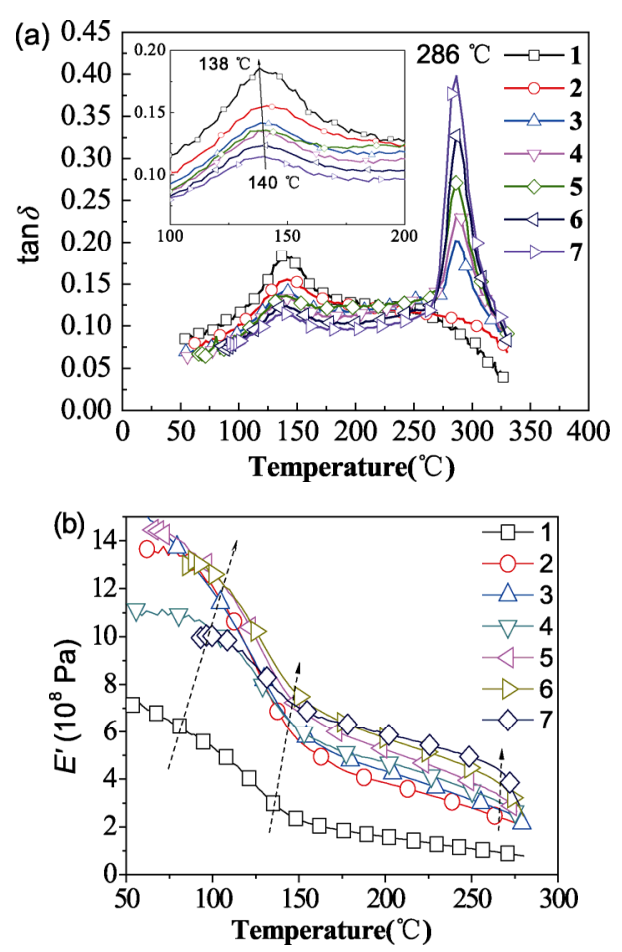

Fig. 4 (a) Loss factor and (b) storage modulus in the DMTA of PTFE, BN/PTFE, and BN/PI/PTFE.

PI improves the thermal deformation-resistance of PTFE.

The storage modulus of PTFE and its composites decreases due to softening when the specimens are heated up. In Fig. 4(b), the low-temperature inflection point for the PTFE phase shifts higher in temperature. Another inflection point for the PI phase almost remains constant. More importantly, the filling of BN into PTFE boosts the elastic modulus of the composite. This upward trend continues upon the introduction of PI in BN/PTFE, and such increment goes on as the amount of added PI increases. These results demonstrate that the heat-resistance and elastic recovery of PTFE are enhanced by filling it with $\mathrm{BN}$ and by blending it with PI.

It should be noted that the bulk component in the composites is crystalline PTFE. The crystallinity of PTFE decreases when the amounts of BN and PI in composites are increased, which is exemplified by the smaller melting peaks in Fig. 5(a) and the smaller crystallization peaks in Fig. 5(b) for the composites with higher concentrations of BN and PI.

Heterogeneous nucleation and rapid crystallization of PTFE occurs at the interfaces of $\mathrm{BN}$ and PI
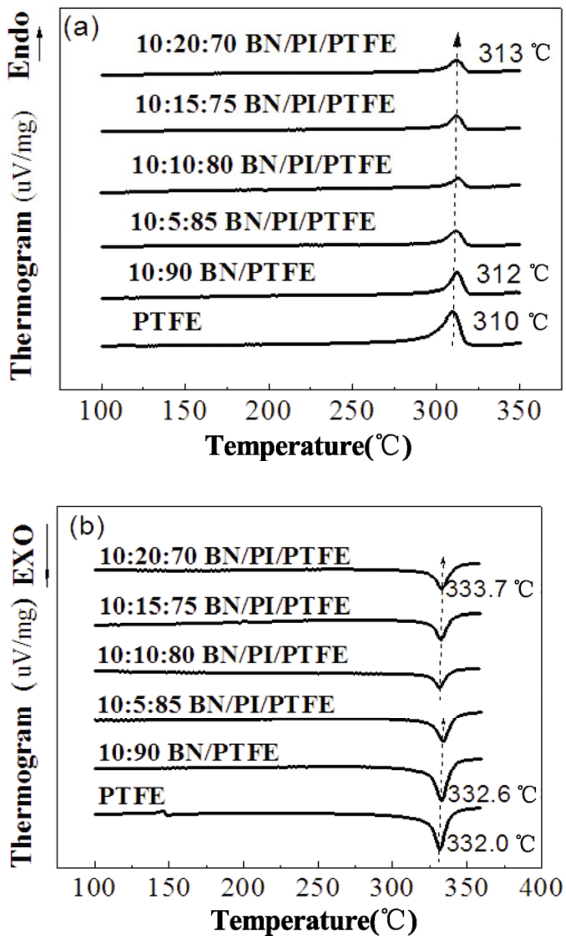

Fig. 5 DSC thermograms of PTFE, BN/PTFE, and BN/PI/PTFE for (a) cooling and (b) reheating.

domains. Thus, the cooling peak shifts to a slightly higher temperature. The peak crystallization temperature varies from $310{ }^{\circ} \mathrm{C}$ for PTFE to $312{ }^{\circ} \mathrm{C}$ for $\mathrm{BN} / \mathrm{PTFE}$ and further up to $313{ }^{\circ} \mathrm{C}$ for BN/PI/PTFE, as shown in Fig. 5(a). The increment is also accompanied by a decrease in the peak width.

As shown in Fig. 5(b), the melting points of the quenched specimens slightly shift towards a higher temperature when BN and PI are mixed into PTFE. This may be due to the greater crystalline grain of the PTFE component, although its content is gradually reduced in the composites.

PTFE has a low thermal conductivity coefficient of $0.24 \mathrm{~W} /(\mathrm{m} \cdot \mathrm{K})$, as shown in Fig. 6. The addition of the good heat-conductor $\mathrm{BN}$ in $\mathrm{BN} / \mathrm{PTFE}$ significantly improves it to 0.66 , which is nearly three times that of PTFE. The added PI further enhances heat transfer at the phase interfaces, as illustrated by a slight increase in the thermal conductivity coefficient. These results are expected when employing $\mathrm{BN}$ as a lubricating filler and PI as its adhering agent with PTFE, as they aid in the timely conduction of the heat generated at the surface during friction to reduce the temperature of the seal and avoid softening failure. 


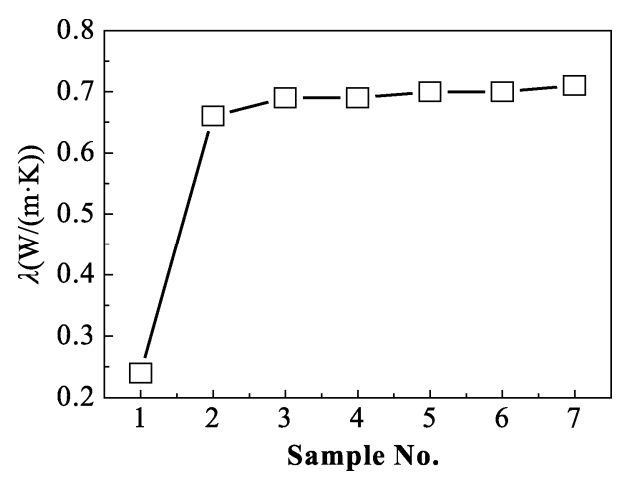

Fig. 6 Thermal conductivity coefficients of PTFE, BN/PTFE, and $\mathrm{BN} / \mathrm{PI} / \mathrm{PTFE}$.

\subsection{Friction and wear properties of composites}

The influences of $\mathrm{BN}$ and $\mathrm{PI}$ on the resultant composites with the PTFE matrix are shown in Fig. 7. The COF increases with time under a load of $200 \mathrm{~N}$ and a rotation speed of $200 \mathrm{rpm}$ for the dry friction of specimens against a metal ring at the ambient temperature of $25{ }^{\circ} \mathrm{C}$, as illustrated in Fig. 7. Usually, the COF substantially rises from zero at the beginning to a maximum, at which the relatively loose pits and budges on the specimen surface become closely compressed against friction. Thereafter, the COF decreases when the surface is smoothened until adhesion occasionally occurs at the general contact area. The undulation then continues until the adhered local protruding portions are torn off or the abrasive fragments are stricken away. Multiple smaller summits and valleys appear due to similar mechanisms in the local regions.

The PTFE resin is mainly assembled by the van der Waals forces between soft molecular segments, which undergo sliding upon loading. The friction heat further induces thermal-mechanical deformation. As previously discussed, BN and PI improve the hardness, heat-conductivity, as well as the thermalmechanical deformation resistance, of PTFE. Thus, the COF of all of the composites is lower than that of PTFE, confirming the effectiveness of the modifications.

Differences are observed in the modifications of the specimen surface layer when BN was filled and PI was blended into PTFE. The rigid BN reinforces and lubricates PTFE. The increase in the bonding of BN with PTFE by the addition of a moderate amount of PI markedly decelerates the initial rapid increase of friction and lowers the maximum COF. However, the enhancement caused by BN is offset during the final stable friction stage when PI covers the BN particles. This explains why the final COF of BN/PI/PTFE is often higher than that of BN/PTFE. Meanwhile, the complex combinations of their effects render a complicated behavior during the intermediate friction stage, as shown in Fig. $7(b)$.

The average of the COF fluctuation towards a constant value in Fig. 7 is taken as the representative value listed in Fig. 8(a). It is less than 0.18 for the composites, which is only $80 \%$ of that of pure PTFE. The preferential concentration of PI and BN in the composite is $5 \%-15 \%$ and $10 \%$, respectively.

The COF influences the running power of the sealing specimen against the coupling counter. Wear resistance is the embodiment of material durability. The specific wear rate of PTFE is $851 \times$ $10^{-6} \mathrm{~mm}^{3} /(\mathrm{N} \cdot \mathrm{m})$, which is far higher than that of the composites in Fig. 8. The wear loss of PTFE reduces upon filling with $\mathrm{BN}$ and decreases further upon blending with PI. The degree of reduction rises with
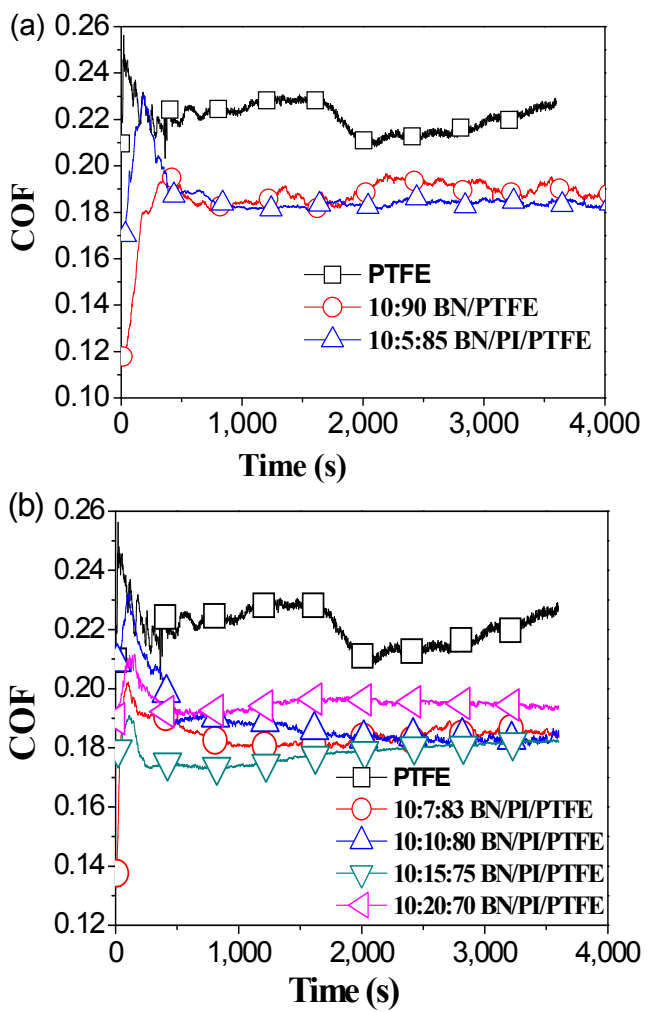

Fig. 7 Evolution of the COF of PTFE, BN/PTFE, and BN/ PI/PTFE. 

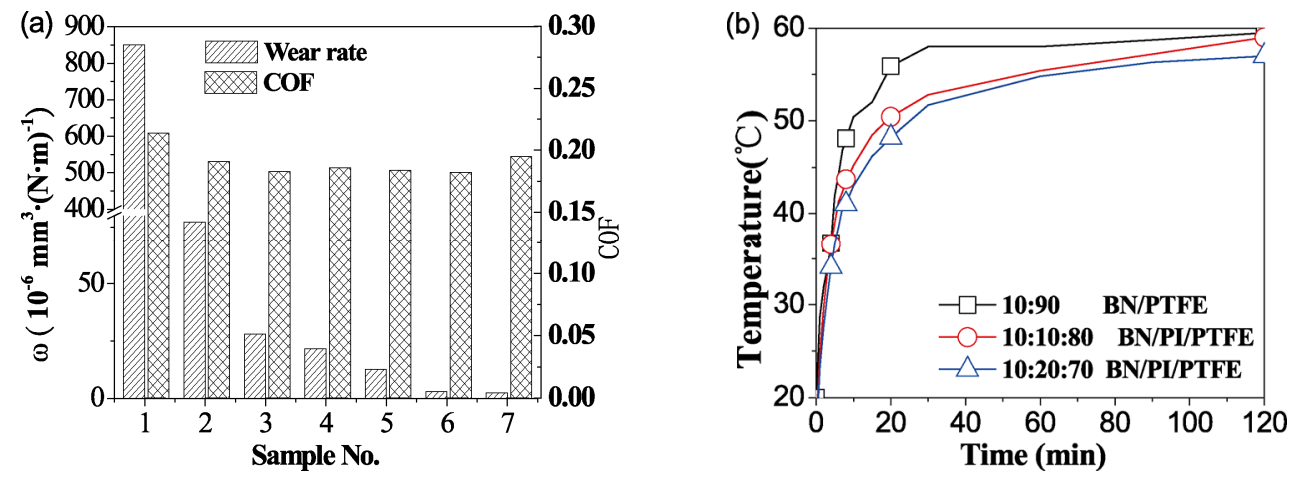

Fig. 8 (a) COF and specific wear rate of specimens and (b) temperature evolution.

the amount of PI added. The wear rate of the 10: $15: 75 \mathrm{BN} / \mathrm{PI} / \mathrm{PTFE}$ composite is $2.9 \times 10^{-6} \mathrm{~mm}^{3} /(\mathrm{N} \cdot \mathrm{m})$, which is nearly $1 / 300$ of that of pure PTFE, nearly $1 / 27$ of that of the 10:90 BN/PTFE composite, and $1 / 10$ of that of PI or graphite modified PTFE. In other words, BN and PI both effectively contribute to improving the wear-resistance of PTFE by enduring shear and plastic deformation.

In friction, wear is accompanied by the accumulation of heat, as demonstrated by the temperature rise in Fig. 8(b). The rapid increase is followed by a gradual increase up to stable value, which is realized when the increment in friction heat is instantaneously transferred into the surrounding air. The small differences between the curves can be explained by the fact that a higher thermal conductivity coefficient is favorable for heat transfer when the produced heat is almost the same as that produced with the fixed operational parameters of $200 \mathrm{~N}$ and $200 \mathrm{rpm}$. A higher sliding velocity and larger normal load result in a higher amount of mechanical energy that transforms into heat, resulting in a higher temperature.

\subsection{Wear mechanisms of the composites}

The surface structure of the samples partially uncovers the wear mechanisms, as seen in the microscopic image (Fig. 9) of the worn surface of PTFE and composite specimens.

For the PTFE specimen, pits due to peeling and flake stains due to the re-bonding of the worn chippings are observed on the worn smooth surface in Fig. 9(a), which indicates the adhesive wear mechanism when the relative soft PTFE comes in contact with the hot metal ring after friction. Inevitably, the local softened PTFE flakes torn from the specimen are transferred onto the metal ring surface, which lubricates the sliding contact faces, thereby decreasing friction.

In the BN/PTFE composite, the rigid $\mathrm{BN}$ particles are trapped in the PTFE matrix, as shown in Fig. 9(b). Some protruding portions of the particles can protect the PTFE matrix through the lubrication of the hardened surface, decreasing the friction on the sliding contact faces. Here, the abrasive wear mechanism plays a significant role. It should be noted that friction on the bulged regions of the PTFE matrix occurs in an adhesive wear manner against the lubricating layer on the metal ring. Some BN particles are torn off from the specimen and migrate towards the metal friction ring. Under the vertical friction load, some of the abrasive chips are pressed and stuck onto the metal surface. In turn, the chips rub or plough the specimen forming furrows on its worn surface in the direction of friction. This is clearly shown in Fig. 9(b).

When PI is blended into PTFE, the dispersed domains of the former are strongly bonded to the latter, even covering some of the $\mathrm{BN}$ particles, as shown in Fig. 9(c). The wear strength of PI is very high. In friction, the torn flakes are smaller than the reduced bulk shedding. The furrows along the direction of friction on the wear surface of the $\mathrm{BN} /$ PI/PTFE composites become shallower than those on BN/PTFE, as shown in Figs. 9(c) and 9(d). Moreover, many micro-convexes and sunken dents block the furrows. The hardened surface of the composite specimens with a low content of PI (i.e., not higher than $10 \mathrm{wt} \%$ ) undergoes friction through the abrasive wear mechanism. The effects of coating the BN particles and the reinforcement of PTFE by PI are remarkable, as demonstrated in Fig. 9(d). 

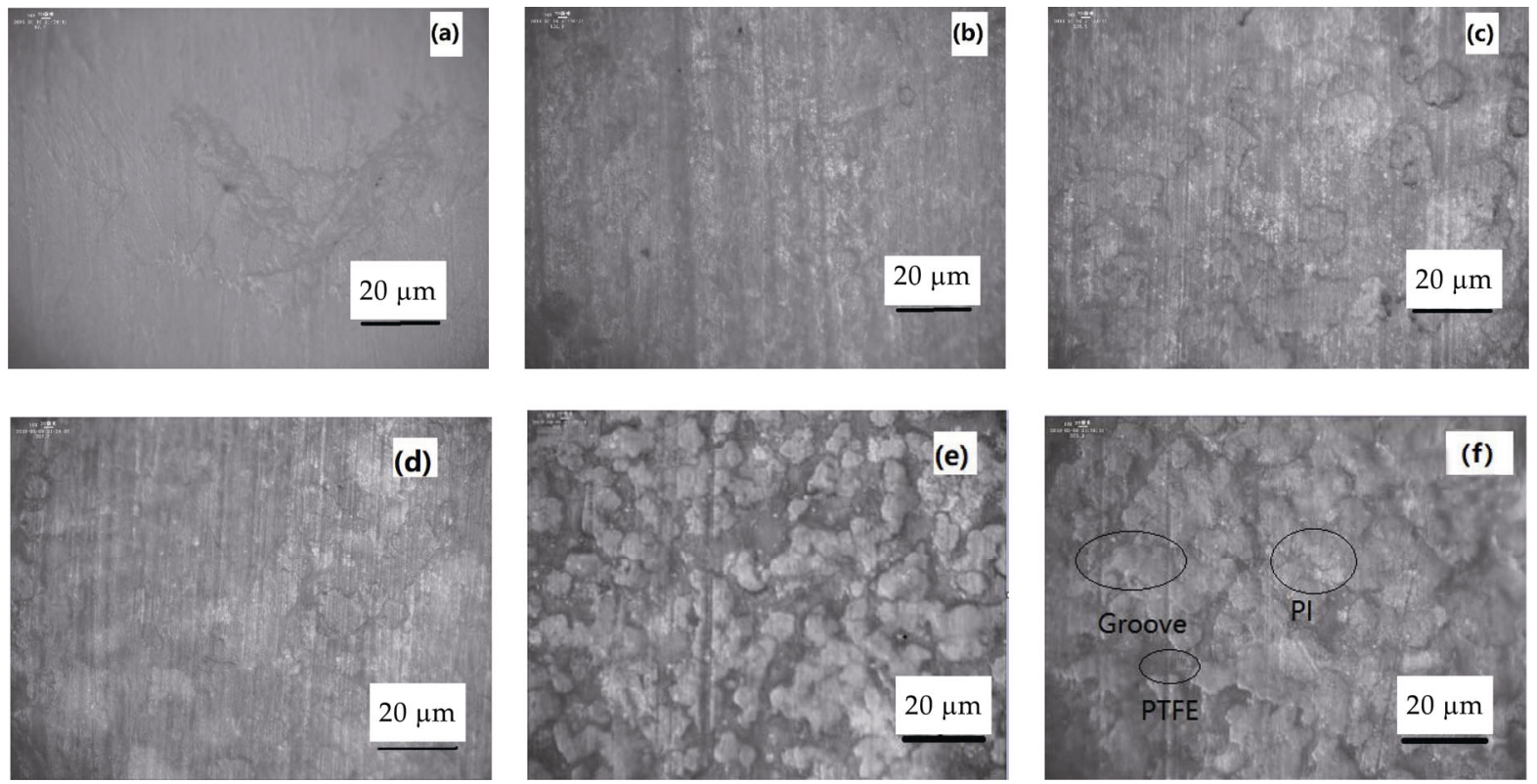

Fig. 9 Microscopic images of the worn surfaces of (a) PTFE, (b) 10:90 BN/PTFE, (c) 10:5:85 BN/PI/PTFE, (d) 10:10:80 BN/ PI/PTFE, (e) 10:15:75 BN/PI/PTFE, and (f) 10:20:70 BN/ PI/PTFE.

At high concentrations of PI, it is inclined to aggregate in the composite. Harder PI domains share the friction shear and normal pressure with the relatively softer PTFE, reducing the total wear loss. In contrast, the resulting heavily uneven phase boundary reinforced by the $\mathrm{BN}$ particles increases the sliding obstruction responsible for the higher COF of the composite with $20 \mathrm{wt} \%$ of PI. Some scars due to the de-bonding of the broken PI heaping occurs on the sample surface. Again, adhesive wear is more predominant than abrasive wear. The fretted debris and aggregation can be observed in Fig. 9(e). The continuous collapsed lumps and stick-slip furrows are present in Fig. 9(f).

The static flat specimen is closely compressed onto the rotating friction ring such that the circular contact area gradually rises during the wear tests, as shown in Fig. 10. The relatively soft PTFE is adhesively worn off; hence the high COF and the severe weight loss are obtained during the wear test. For the hardened PTFE filled with BN, the rough grains are abrasively rubbed away as they are randomly dispersed and embedded within the PTFE matrix with certain agglomerations, and the composite still inherits some adhesiveness. In these two cases, friction and wear-resistance are simultaneously improved. In the third case, the interpenetrated blend structure with the stronger PI reduces the high ratio of the adhesive wear of the relatively weaker PTFE matrix. In the fourth case, $\mathrm{BN}$ further reinforces and lubricates the PI/PTFE blend. This occurs through the screen-meshing of the $\mathrm{BN}$ particles in dispersed interpenetrated domains or small interlocked networks at the molecular level when the partially compatible PI is blended with PTFE because of the stronger bonding of $\mathrm{BN}$ with PI than with PTFE. Two aspects of the tribological properties can both be optimized in the threecomponent composites with a low PI content. However, the coarseness due to the aggregated PI domains in the surface layer requires higher friction in sliding for the composites with a high PI content. Collapse likely occurs during wear when the PI and BN contents are higher than ca. $10 \mathrm{wt} \%$ and $15 \mathrm{wt} \%$, respectively. The transition from the abrasive wear mechanism to the adhesive wear mechanism is observed. The transition depends not only on the material composition and microstructure but also on friction conditions, such as the sliding velocity and normal loading, as well as the lubrication medium. This is partially confirmed by the observed microstructures. 


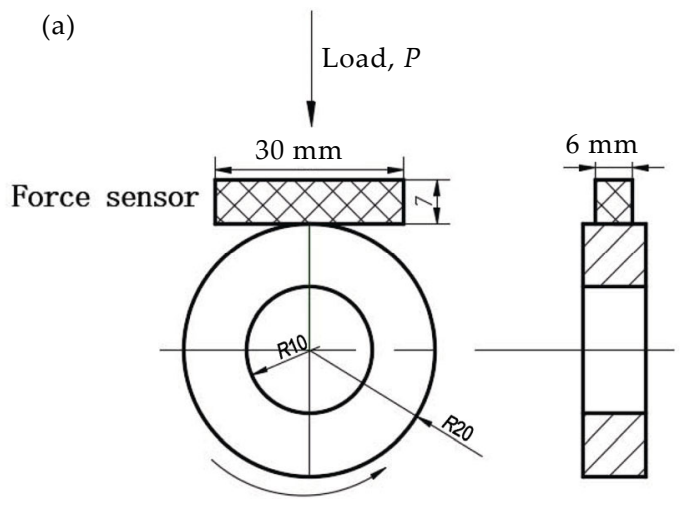

(b)
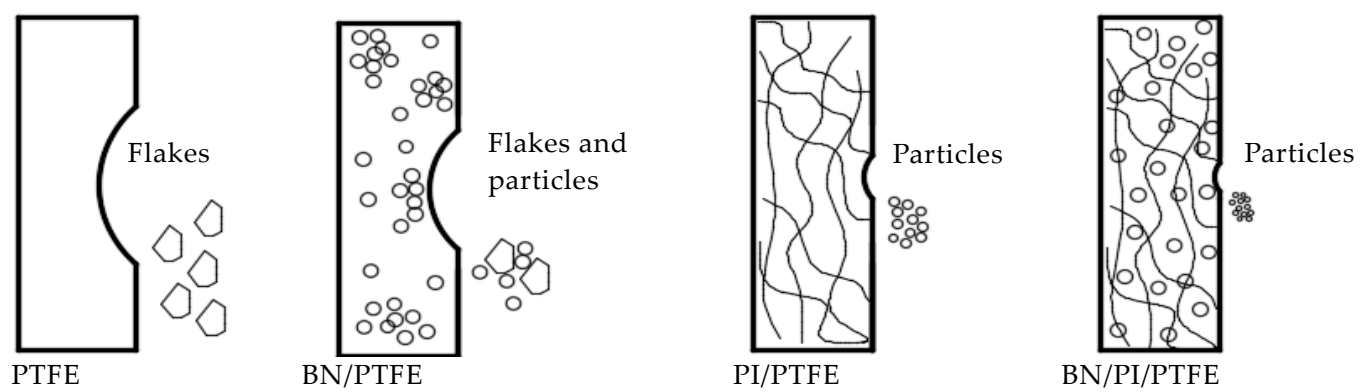

Fig. 10 Schematics of (a) friction test and (b) wear mechanisms of PTFE and composites.

Based on the preliminary results, the following analyses were conducted considering the characteristics of each component.

\subsection{Effects of friction conditions on the wear of 10:15:75 BN/PI/PTFE composites}

The influences of sliding velocity and external loading on friction and wear under a dry ambient condition at a room temperature of $25{ }^{\circ} \mathrm{C}$ are shown in Fig. 11. The evolutions of the COF with respect to the load and rotation are shown in Figs. 11(a) and $11(\mathrm{~b})$, respectively. The total wear rate and the resultant temperature rise are shown in Figs. 11(c) and $11(\mathrm{~d})$, respectively. The temperature is only indicative of the degree of friction-heating and does not determine it, because of a portion of the heat transfers to the cool air or the instrument.

At a ring rotation speed of $200 \mathrm{rpm}$ and a test duration of $2 \mathrm{~h}$, the COF rapidly increases and then drops, as shown in Fig. 11(a). Thereafter, the curve rises with some fluctuations and terminates at a lower value for the tests conducted at a low loading of 50-200 $\mathrm{N}$ when almost remaining constant at a high loading of $300-400 \mathrm{~N}$. The COF decreases with the increasing friction load except at the highest load of $400 \mathrm{~N}$. The variation in the amplitude of fluctuation with loading has similar behavior. It is noted that the reduction in the COF and fluctuation amplitude corresponds to a lower wear rate in Fig. 11(c) and a higher temperature rise in Fig. 11(d) when the load increased.

According to the Coulomb friction model, the $\mathrm{COF}$ is proportional to the normal load in regular sliding friction. However, irregular cases can occur such as those in Fig. 11. The COF is 0.25 at $50 \mathrm{~N}$ but 0.15 at $300 \mathrm{~N} / 200 \mathrm{rpm}$. This indicates the notintimate contact between the composite specimen and its opponent in friction at a low loading of $50 \mathrm{~N}$, where the abrasive wear exceeds the adhesive wear, with friction collision inducing the fluctuation. The hard ridges on the steel rotator elastically scratch the composite surface, causing micro-cutting, ploughing, and the subsequent local delamination. The resultant rougher specimen surface induces a higher COF with time, as shown in Fig. 11(a). In addition, the stick-slip is easily generated and eventually results in frictional vibration, as represented by the fluctuation noise in the COF curve. 

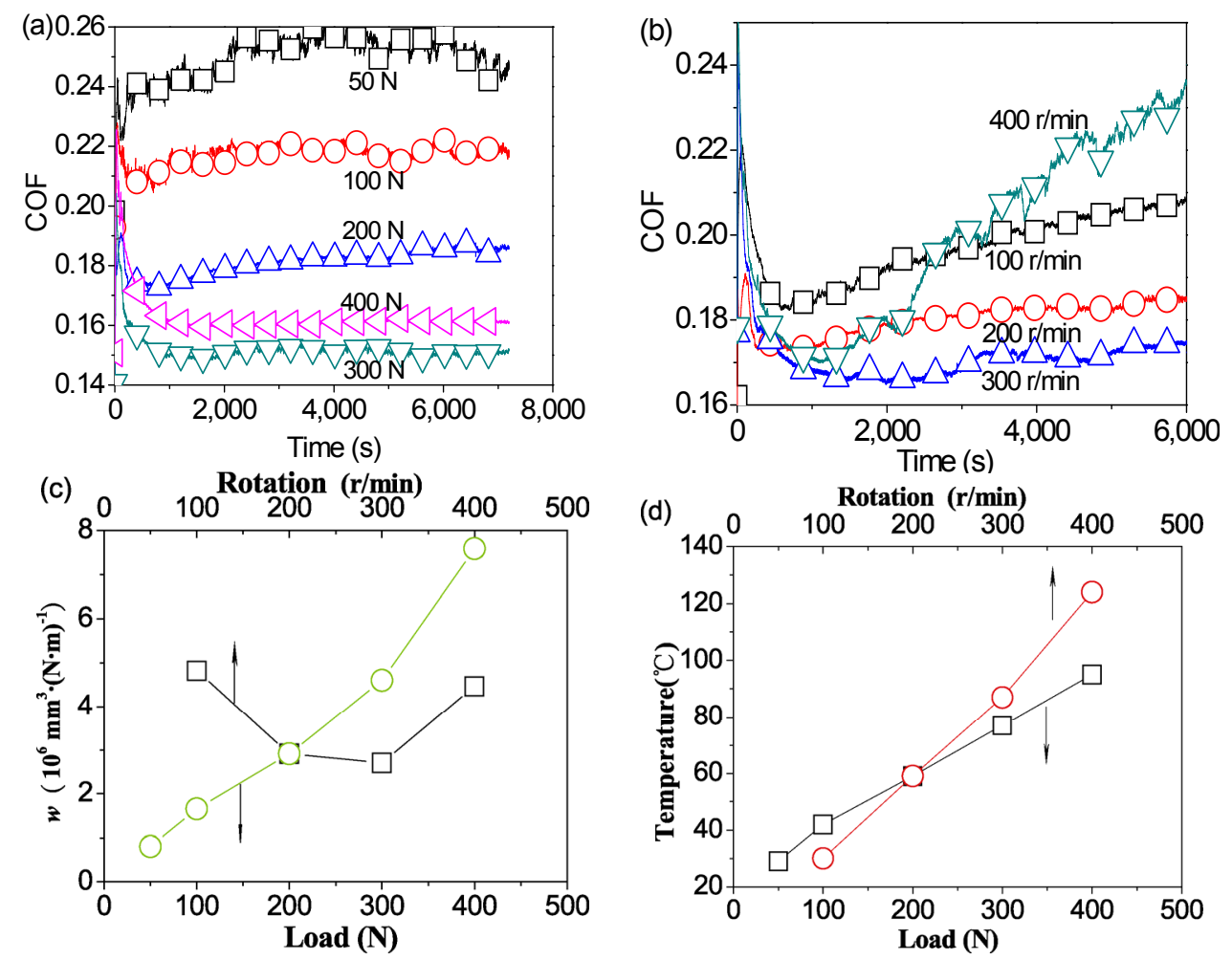

Fig. 11 Effects of (a) load and (b) rotation on the COF versus time, on (c) the volume wear rate, and (d) the final temperature of the 10:15:75 BN/PI/PTFE composites.

When a larger normal pressure is applied to the specimen, a greater number of bumps are collided off, increasing the contact area in favor of forming a transfer film on the rotator. The total adhesive loss becomes larger, in contrast with the lower
COF, due to such lubrication at higher loads of 200-300 N. This is confirmed from the observation that the furrows of the abrasive surface become shallower and more flattened as the load increases from 50 to $300 \mathrm{~N}$ (Figs. 9(e), 12(a), and 12(b)).
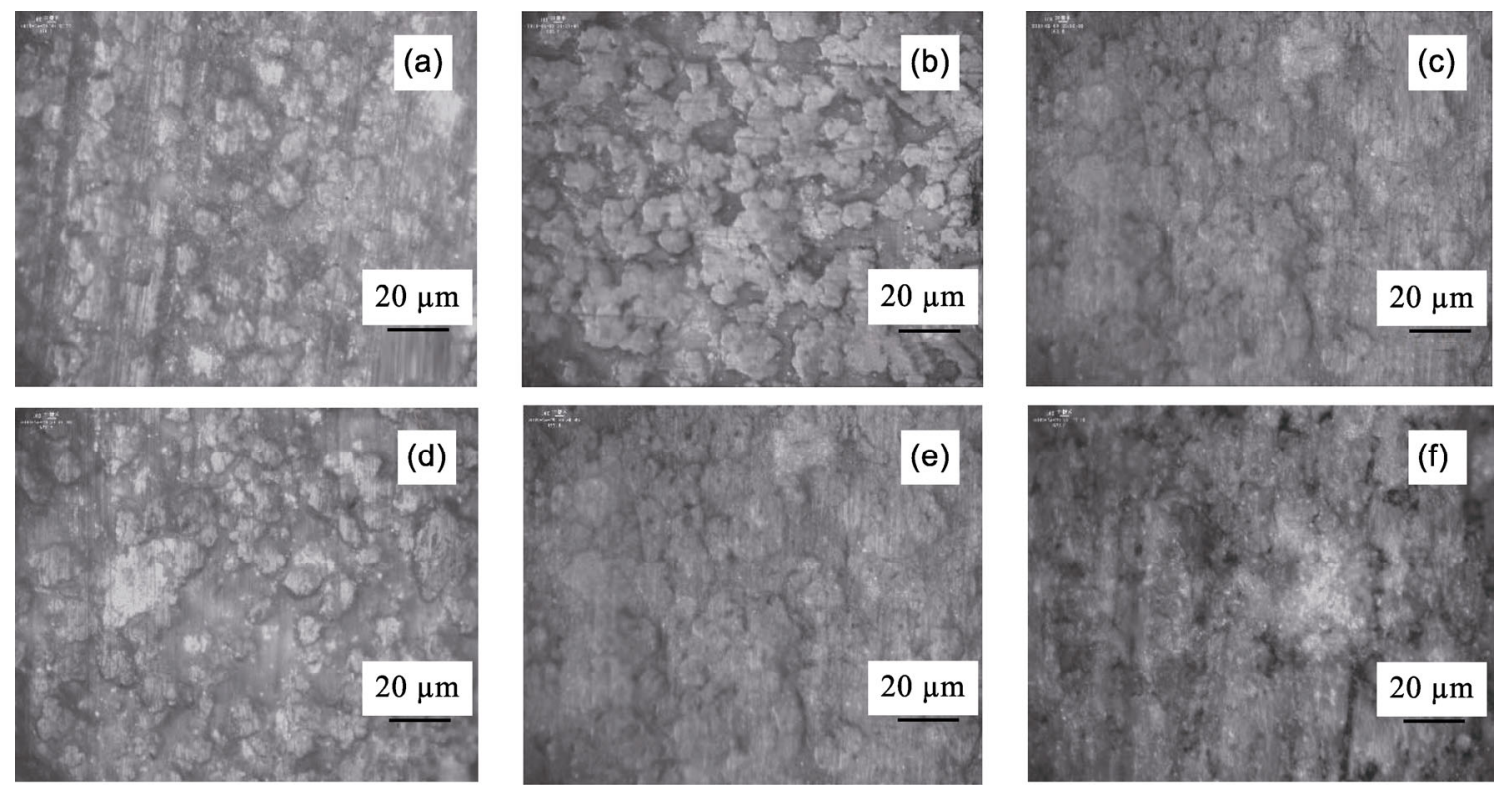

Fig. 12 Microscopic images of the surface of 10:15:75 BN/PI/PTFE worn at $200 \mathrm{rpm}$ and (a) $50 \mathrm{~N}$, (b) $300 \mathrm{~N}$, and (c) $400 \mathrm{~N}$; at $200 \mathrm{~N}$ and (d) $100 \mathrm{rpm}$, (e) $300 \mathrm{rpm}$, and (f) $400 \mathrm{rpm}$. 
The close-intimate contact is realized when the load is augmented to $400 \mathrm{~N}$, where adhesive wear is dominant, resulting in a larger wear rate when the specimen is plastically deformed or even delaminated by stronger heating. The dispersed domains dissociate to be semi-continuous by the forced plastic shear, crack nucleation and propagation, and displacement of material within the anomalous grooves on the specimen surface worn at $400 \mathrm{~N}$, as shown in Fig. 12(c). The composites become softer as indicated by the high surface temperature at higher loads, as shown in Fig. 11(d). The maximum specific wear rate of $1.5 \times 10^{-5}$ $\mathrm{mm}^{3} /(\mathrm{N} \cdot \mathrm{m})$ at $400 \mathrm{~N}$ is nearly 76 times more than the minimum of $2 \times 10^{-7} \mathrm{~mm}^{3} /(\mathrm{N} \cdot \mathrm{m})$ at $50 \mathrm{~N}$.

At the moderate load of $200 \mathrm{~N}$, within $2 \mathrm{~h}$, the COF varies with time, as shown in Fig. 11(b). The COF decreases with increasing friction rotation speed except at the highest speed of $400 \mathrm{rpm}$. With respect to friction, the larger the rotation speed (below $300 \mathrm{rpm}$ ) is, the less the abrasive loss of the specimen; hence, the smaller the COF and wear rate are due to surface smoothening for the composite softened by the accumulated frictional heat and surface slip. More heat is accumulated as indicated by the high surface temperature at higher rotation speeds, as shown in Fig. 11(d). The thickened soft transfer film formed on the friction ring lubricates the contact surface. The specific wear rate is then reduced from $4.8 \times 10^{-6} \mathrm{~mm}^{3} /(\mathrm{N} \cdot \mathrm{m})$ at $100 \mathrm{rpm}$ to $2.7 \times 10^{-6} \mathrm{~mm}^{3} /(\mathrm{N} \cdot \mathrm{m})$ at $300 \mathrm{rpm}$. However, the overheated surface can become soft or even sticky at a rotation speed as high as $400 \mathrm{rpm}$. This induces the adhesive wear or even the eventual surface collapse due to the local melting corresponding to the rapid increase in the COF. The specific wear rate suddenly rises to $4.46 \times 10^{-6} \mathrm{~mm}^{3} /(\mathrm{N} \cdot \mathrm{m})$, corresponding to a contact temperature close to the glass transition temperature. The images of the specimen surfaces worn at 100, 200, 300, and 400 rpm are respectively characterized by broken spot defects in Fig. 12(d), furrows surrounded by domain aggregates in Fig. 9(e), a glutinous texture in Fig. 12(e), and deep grooves locally peeled into dents in Fig. 12(f). The high friction force contributes to the severe wearing of the domain dumplings due to the strong shearing of the junctions formed by the adhesion between the contacting surfaces and due to the subsequent plastic deformation or softening caused by the dissipation of energy. In a long-term operation, micro-cracking and collapse can be induced by fracture and fatigue.

\subsection{Wear transition maps of BN/PI/PTFE composites}

For the soft to hard mating interfaces of PTFE against steel, a consistent law for friction and wear, which identifies the concrete mechanisms, so far, has not yet been established despite the classification of the relative factors [49], the summary of the relative factors [50], and the artistic description of the wear map [39]. Hence, the mapping analyses below are simply explored based on the foregoing observations.

It is worth noting that wear is complicated, as it is a multi-parameter and sensitive process in the friction of composites. As Sahin [49] pointed out, the wear mechanism and wear rate depend on several aspects, such as the friction material (matrix, reinforcement, interface, composition, and texture), counterpart surface (asperity, hardness, toughness, particle geometry, and distribution), relative movement of friction couple (sliding, rolling, fretting, and impact, etc.), test parameters (load, velocity, temperature, and contact situation), and environmental conditions (humidity, corrosive medium, gas or liquid phase, and complexity). The coupling of these factors results in the different wear modes of abrasive, adhesive, fatigue, diffusive, and their combinations [51]. Similar to the evolution of the COF, as shown in Figs. 7 and 11, the material state generally varies with time underwear parameters in spatial conditions, as implied in Fig. 13, which can be referred to as the wear sphere. Under such a generalized framework,

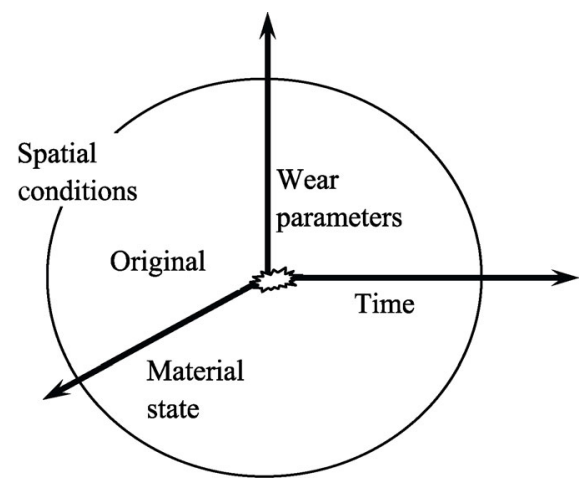

Fig. 13 Wear sphere in the evolution of frictional material and parameters in time and space. 
the wear mechanisms and their transitions can be further concluded by examining the successive changes in the microstructure and the microgeometry of the contact surfaces.

\subsubsection{Wear transitions of BN/PI/PTFE at $100 \mathrm{rpm}$ under $200 \mathrm{~N}$}

At the moderate running conditions of sliding speed and normal pressure (100 rpm and $200 \mathrm{~N}$ ), the specimens almost remain unchanged in terms of the surface temperature (below $60{ }^{\circ} \mathrm{C}$ ), as shown in Fig. 8 . The material properties play a significant role in friction. The wear rate results are shown in Fig. 14(a).

The wear map can be drawn as the COF versus the PI content, as shown in Fig. 14(b). The wear transition map by composition reflects the working condition and material characteristics. The transitions from adhesive wear to abrasive-adhesive wear, further to adhesive-fretting, and finally to adhesive-stick slip wear are observed at a low to medium COF with the incorporation $\mathrm{BN}$ and the further addition of PI into

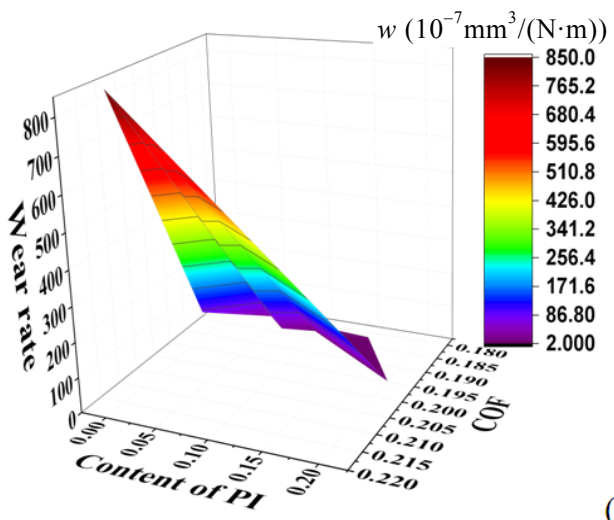

(a)
PTFE, as shown in Fig. 9, because of the coating and lubrication caused by $\mathrm{BN}$ and the reinforcement of PTFE with PI in the composites. The transitions may be extended to higher PI contents with reducing COF. For the harder and rougher BN/PTFE and 10:5:95 $\mathrm{BN} / \mathrm{PI} / \mathrm{PTFE}$, severe abrasive wear is possible with a high COF. In contrast, the composites with a high PI content (15-20 wt\%) predominately fall into the adhesive mode, accompanied by fatigue or other modes of wear, as indicated by the lower COF. This wear rate map of the COF versus composition helps choose a material, in which the appropriate percentage of PI is in the range of $10 \mathrm{wt} \%-15 \mathrm{wt} \%$ for mild wear.

\subsubsection{Wear and friction of 10:15:75 BN/PI/PTFE under different pressures and sliding speeds}

For the BN/PI/PTFE composite with one optimal composition of 10:15:75, the wear rate strongly depends on the working parameters, as shown in Fig. 15(a). Its wear rate is plotted concerning the

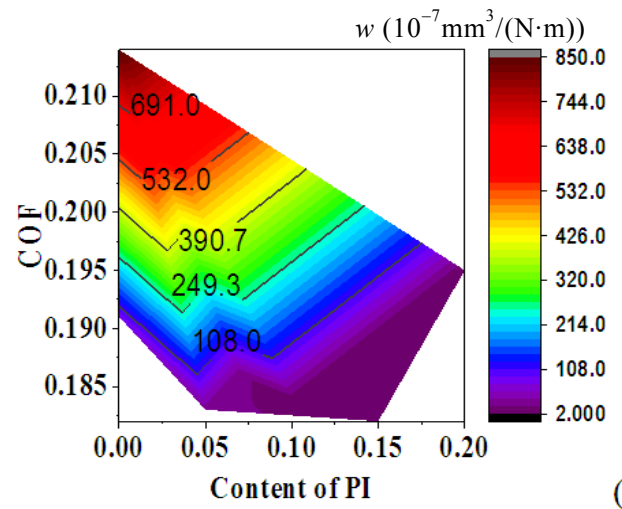

(b)

Fig. 14 Wear rate: (a) friction plot and (b) wear map by composition at $200 \mathrm{~N}$ and $100 \mathrm{rpm}$.
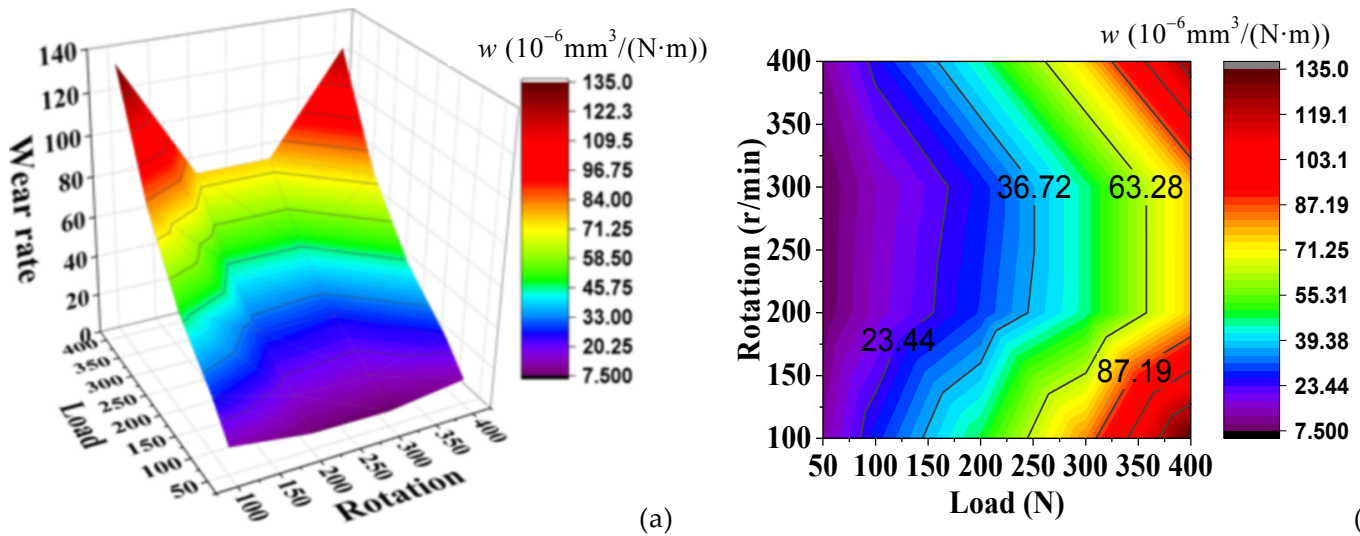

Fig. 15 (a) Wear plot and (b) wear transition map of 10:15:75 BN/PI/PTFE. 
rotation speed and the normal load, as shown in Fig. 15(b), and the plot is called the wear mechanism transition map. The map can be usually divided into four regimes based on the severity of wear considering the worn surfaces: (a) Very mild wear (wear rate $\leqslant 2 \times 10^{-5} \mathrm{~mm}^{3} /(\mathrm{N} \cdot \mathrm{m})$ ), (b) mild wear $(2 \times$ $10^{-5}<$ wear rate $\left.<4 \times 10^{-5} \mathrm{~mm}^{3} /(\mathrm{N} \cdot \mathrm{m})\right)$, (c) medium wear $\left(4 \times 10^{-5}<\right.$ wear rate $\left.<7 \times 10^{-5} \mathrm{~mm}^{3} /(\mathrm{N} \cdot \mathrm{m})\right)$, and (d) severe wear (wear rate $\geqslant 9 \times 10^{-5} \mathrm{~mm}^{3} /(\mathrm{N} \cdot \mathrm{m})$ ).

The transitions along the load axis at a certain rotation speed occur sequentially from the light abrasive wear at a low load, the coexistent abrasiveadhesive wear at a secondarily mild load, the adhesiveadhesive wear with fretting at a medium load, and finally to the strong adhesive wear with crack and groove at a high load. However, the transitions along the rotation speed axis completely depend on the load level. In Figs. 12(d)-12(f), at a fixed load of $200 \mathrm{~N}$, the sequential wear mechanisms are spot-adhesive, adhesive plus abrasive, abrasive, and strip-adhesive plus abrasive, as the sliding speed increases.

\subsubsection{Monitoring of friction and wear}

The above transitions in wear mechanisms mainly depend on the variations in the thermal-mechanical state and surface structure of the composites due to compression/shear and the accumulated friction heating. The COF can be calibrated from the plot of the rotation torque versus the constant loading weight. The surface temperature of the specimen can be directly measured using an uncontacted thermometer. Thus, the friction and wear state under a certain normal pressure can be indirectly predicted and monitored using the sliding speed (transformed from rotation) and temperature.

For the 10:15:75 BN/PI/PTFE composite specimen against the counter-surface, the sliding COF varies with the compressive load and rotation speed, as shown in Fig. 16(a). Its contour curves correspond to the friction transition maps in Fig. 16(b). The four general regimes are impact and abrasive wear on a small contact area at a low load, abrasive sliding and fretting at a mild load, adhesive sliding by lubrication due to the transfer film at a medium load, adhesive sliding through plastic deformation, and debris rolling along the load axis at a certain rotation. To conserve mechanical work under a low $\mathrm{COF}$, the intermediate sliding velocity is better kept as it is usually operated in most practices. Grooves are often observed on the worn surface caused by strong wear at a high velocity, instead of plowing, dents/ridges caused by impact, and a subsequent delamination at a low velocity under a low normal load.

Distinctly different from the hard to hard mating surfaces, the coupling of the soft PTFE composite with hard steel is more sensitive to temperature. Temperature rise is both the consequence and the cause for the varied developments of friction and wear. Operating under a high load and high rotation speed results in a higher temperature, as shown in Fig. 17(a), and the differential wear transitions are presented in Fig. 17(b). The variation of wear transition due to temperature is due to the elastic, elasto-plastic, and plastic wear modes along the temperature axis, or descriptively in the thermo-mechanical, mechanical, and thermal wear types along the load axis.
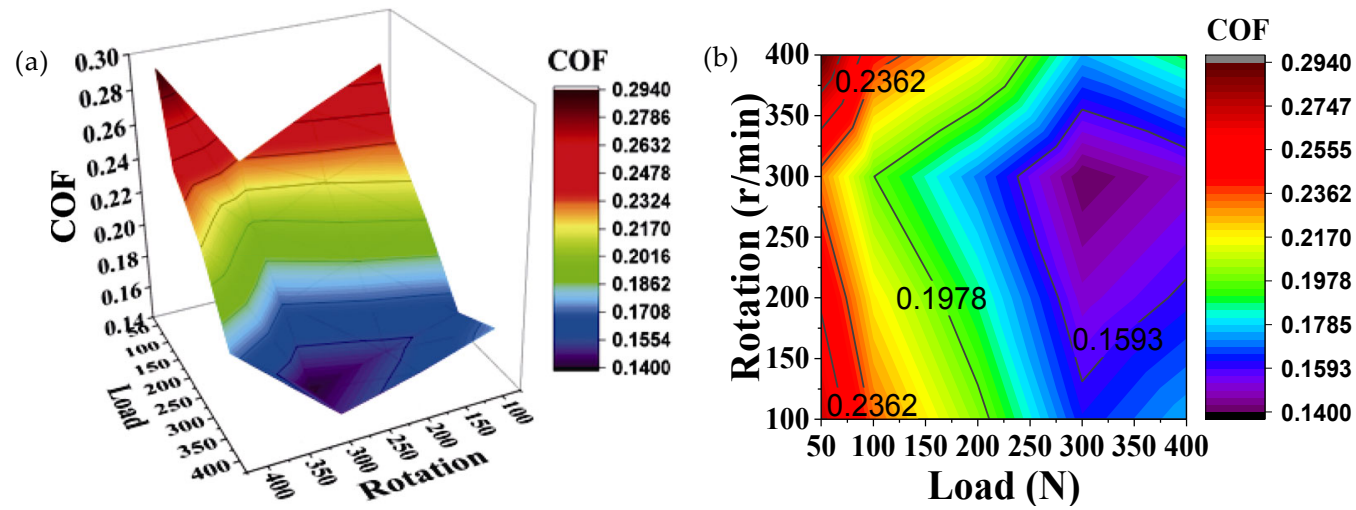

Fig. 16 (a) Friction plot and (b) friction transition map of 10:15:75 BN/PI/PTFE. 

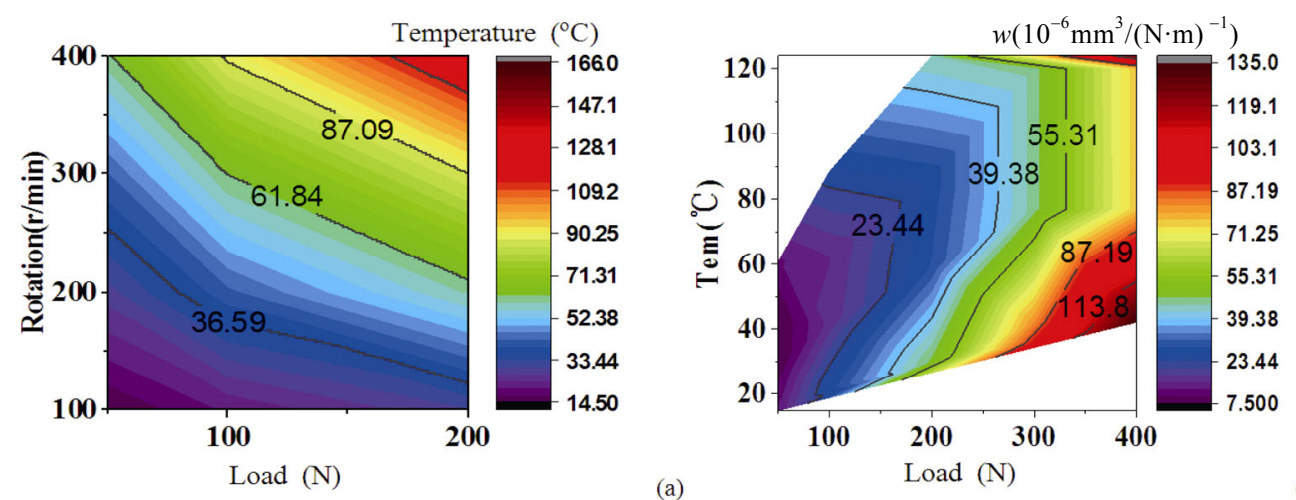

(a)

(b)

Fig. 17 (a) Friction temperature map and (b) wear transition of 10:15:75 BN/PI/PTFE.

The contact areas and transfer film during wear strongly depend on the test parameters and the sample materials. Samyn and Schoukens [52] pointed out that the product of pressure (or normal load) and the sliding velocity reflects the mechanical work dissipation. Thus, the area distribution is representative of the mechanisms in terms of wear, friction, and thermal state, as shown in Figs. 15(b), 16(b), and 17(a), respectively. The formed debris frets include plastic deformations due to long-term friction [53], and more wear debris can cause three-body wear [54]. At high temperatures, the sample surface softens, whereas the wear debris becomes easily susceptible to slip and dynamic adhesiveness, aggravating the tear. From the friction temperature map (Fig. 17(a)) and the wear rate map (Fig. 17(b)), it can be seen that the contact sample undergoes from the elastic to viscoelastic then to plastic state [54]. Therefore, it is necessary and possible to simultaneously monitor normal load, pressure, temperature, and sample geometry to characterize the process.

\section{Conclusions}

Remarkably friction- and wear-resistant PTFE composites are successfully prepared by blending the PTFE matrix with PI and filling it with $\mathrm{BN}$ to improve its hardness, thermomechanical storage modulus, thermal conductivity, and tensile behavior, caused by the more superior properties of the additives and their synergistic effects at appropriate concentrations and under moderate conditions.

1) PI can boost the dispersion and bonding of BN to PTFE. The wear rate and COF of the 10:10:80
BN/PI/PTFE composite reduced to almost $1 / 300$ and $80 \%$ of those of pure PTFE, respectively. The appropriate composition induces the partial transition from the dispersed adhesive wear mechanism to the abrasive wear mechanism of PTFE. Apparent agglomeration occurs in high percentages of added PI, which causes adhesive failure.

2) The composites with the optimal composition can be used under a high friction load $(300 \mathrm{~N})$ or at a high rotation speed $(300 \mathrm{rpm})$ in low friction. Severe conditions (e.g., $400 \mathrm{~N}$ and $400 \mathrm{rpm}$ ) result in the accumulation of heat, which induces greater deformations or damage, indicating poor tribological properties.

3) The plots of wear and friction and mechanism transition maps are constructed to understand their relationships with the operating conditions and composite composition under the wear sphere framework.

\section{Acknowledgements}

This work was supported by the National Natural Science Foundation of China (No. 51073021).

Open Access This article is licensed under a Creative Commons Attribution 4.0 International License, which permits use, sharing, adaptation, distribution and reproduction in any medium or format, as long as you give appropriate credit to the original author(s) and the source, provide a link to the Creative Commons licence, and indicate if changes were made.

The images or other third party material in this 
article are included in the article's Creative Commons licence, unless indicated otherwise in a credit line to the material. If material is not included in the article's Creative Commons licence and your intended use is not permitted by statutory regulation or exceeds the permitted use, you will need to obtain permission directly from the copyright holder.

To view a copy of this licence, visit http://creativecommons.org/licenses/by/4.0/.

\section{References}

[1] Gong R, Liu M, Zhang H, Xu Y. Experimental investigation on frictional behavior and sealing performance of different composites for seal application. Wear 324-343: 334339 (2015)

[2] Hu P, Huang C, Zheng H, Li C L, Huang Z C. Research on tribological surface chemistry of PTFE composite. Key Eng Mater 249:481-484 (2003)

[3] Zou A, Li D. Effect of carbon nanotube on the oscillating wear behaviour of metal-PTFE multilayer composites. $J$ Wuhan Univ Technol 33(5):261-264 (2018)

[4] Conte M, Igartua A. Study of PTFE composites tribological behavior. Wear 296(1-2): 568-574 (2012)

[5] Zhang F, Sui T Y, Song B Y, Shang Q, Cao C. The tribological performance research of modified PTFE composite material. Int J Mater Prod Technol 53(2): 101-115 (2016)

[6] Unal H, Mimaroglu A, Kadioglu U, Ekiz H. Sliding friction and wear behaviour of polytetrafluoroethylene and its composites under dry conditions. Mater Des 25(3): 239-245 (2004)

[7] Harris K L, Pitenis A A, Sawyer W G, Krick B A, Blackman G S, Kasprzak D J, Junk C P. PTFE tribology and the role of mechanochemistry in the development of protective surface films. Macromolecules 48(11): 37393745 (2015)

[8] Wieleba W. The role of internal friction in the process of energy dissipation during PTFE composite sliding against steel. Wear 258(5-6): 870-876 (2005)

[9] Mnif R, Ben Jemaa M C, Hadj Kacem N, Elleuch R. Impact of viscoelasticity on the tribological behavior of PTFE composites for valve seals application. Tribol Trans 56(5): 879-886 (2013)

[10] Trabelsi M, Kharrat M, Dammak M. Impact of lubrication on the tribological behaviour of PTFE composites for guide rings application. Bull Mater Sci 39(5): 12051211 (2016)

[11] Subramanian K, Nagarajan R, De Baets P, Subramaniam S, Thangiah W, Sukumaran J. Eco-friendly mono-layered PTFE blended polymer composites for dry sliding tribo-systems. Tribol Int 102: 569-579 (2016)

[12] Nunez E E, Gheisari R, Polycarpou A A. Tribology review of blended bulk polymers and their coatings for high-load bearing applications. Tribol Int 129: 92-111 (2019)

[13] Shen J T, Top M, Pei Y T, De Hosson J T M. Wear and friction performance of PTFE filled epoxy composites with a high concentration of $\mathrm{SiO}_{2}$ particles. Wear 322 323: 171-180 (2015)

[14] Cao W H, Gong J, Yang D Y, Qi Y, Wang H G, Gao G. Tribological behavior of nano-ZrC reinforced polytetrafluoroethylene-polyphenylene sulfide composites. Polym Mater Sci Eng 34(2): 48-55 (2018)

[15] Wang Y, Gong J, Yang D Y, Gao G, Ren J F, Mu B, Chen $\mathrm{S}$ S, Wang $\mathrm{H}$ G. Tribological behavior of nano- $\mathrm{Al}_{2} \mathrm{O}_{3}$ reinforced PPS-PTFE composites. Tribol Trans 57(2): 173-181 (2014)

[16] Wang B H, Lv Q J, Hou G L. Tribological behavior of nano- $\mathrm{Al}_{2} \mathrm{O}_{3}$ and PEEK reinforced PTFE composites. AIP Conf Proc 1794(1): 020030 (2017)

[17] Hong M, Pyun S I. Effect of fluorinated ethylene propylene copolymer on the wear behaviour of polytetrafluoroethylene. Wear 143(1): 87-97 (1991)

[18] Díez-Pascual A M, Díez-Vicente A L. Nano-TiO reinforced PEEK/PEI blends as biomaterials for loadbearing implant applications. ACS Appl Mater Int 7(9): 5561-5573 (2015)

[19] Samyn P, Schoukens G. Tribological properties of PTFEfilled thermoplastic polyimide at high load, velocity, and temperature. Polym Compos 30(11): 1631-1646 (2009)

[20] Zhao G, Wu C H, Zhang L C, Song J F, Ding Q J. Friction and wear behavior of PI and PTFE composites for ultrasonic motors. Polym Adv Technol 29(5): 14871496 (2018)

[21] Rao M, Hooke C J, Kukureka S N, Liao P, Chen Y K. The effect of PTFE on the friction and wear behavior of polymers in rolling-sliding contact. Polym Eng Sci 38(12): 1946-1958 (1998)

[22] Zhang G, Schlarb A K, Tria S, Elkedim O. Tensile and tribological behaviors of $\mathrm{PEEK} /$ nano- $\mathrm{SiO}_{2}$ composites compounded using a ball milling technique. Compos Sci Technol 68(15-16): 3073-3080 (2008)

[23] Sawyer W G, Freudenberg K D, Bhimaraj P, Schadler L S. A study on the friction and wear behavior of PTFE filled with alumina nanoparticles. Wear 254(5-6): 573580 (2003)

[24] Anandarao R K R, Srinivasan S, Rommala H R, Deepthi Y P. Experimental investigation on influence of molybdenum content on tribological properties of hybrid PTFE composite. AIP Conf Proc 2080(1): 020017 (2019) 
[25] Lim W S, Khadem M, Anle Y, Kim D E. Fabrication of polytetrafluoroethylene-carbon nanotube composite coatings for friction and wear reduction. Polym Compos 39(S2): E710-E722 (2018)

[26] Xie S J, Xie W Q. The preparation and performance study of graphene/PTFE composites sealing materials. Lubr Sealing 43(9): 104-107, 140 (2018)

[27] Wang S B, Cao B, Teng B. Torsional tribological behavior of polytetrafluoroethylene composites filled with hexagonal boron nitride and phenyl p-hydroxybenzoate under different angular displacements. Ind Lubr Tribol 67(2): 139-149 (2015)

[28] Park O K, Owuor P S, Jaques Y M, Galvao D S, Kim N H, Lee J H, Tiwary C S, Ajayan P M. Hexagonal boron nitride-carbon nanotube hybrid network structure for enhanced thermal, mechanical and electrical properties of polyimide nanocomposites. Compos Sci Technol 188: 107977 (2020)

[29] Zimmermann-Ptacek J, Muggli M, Wildhack S, Hintzer K, Gerdes T, Willert-Porada M, Moos R. Thermal, dielectric, and mechanical properties of h-BN-filled PTFE composites. J Appl Polym Sci 135(44): 46859 (2018)

[30] Li T L, Hsu S L C. Enhanced thermal conductivity of polyimide films via a hybrid of micro- and nano-sized boron nitride. J Phys Chem B 114(20): 6825-6829 (2010)

[31] Song H, Kim B G, Kim Y S, Bae Y S, Kim J, Yoo Y. Synergistic effects of various ceramic fillers on thermally conductive polyimide composite films and their model predictions. Polymer 11(3): 484 (2019)

[32] Yan Y H,Yang Y L,Jia Z N. Friction and wear behaviors of hybrid PI/PTFE nanocomposites enhanced with nanoparticles. J Balkan Tribol Assoc 22(2A): 17391748 (2016)

[33] Zhao Y L, Qi X W, Zhang W L, Fan B L, Yang Q X. Effects of copper nanoparticles located in different regions of polytetrafluoroethylene/polyimide blends on the morphology, mechanical and tribological properties of PTFE composites. Tribol Lett 67(1): 18 (2019)

[34] Yu C Y, Wan H Q, Chen L, Li H X, Cui H X, Ju P F, Zhou H D, Chen J M. Marvelous abilities for polyhedral oligomeric silsesquioxane to improve tribological properties of polyamide-imide/ polytetrafluoroethylene coatings. $J$ Mater Sci 53(17): 12616-12627 (2018)

[35] Wang J J, Liu T. Tribological properties of polyimide/ graphite modified polytetrafluoroethylene. Polym Mater Sci Eng 34(8): 61-66 (2018)

[36] Momose Y, Kubo K. Observation of triboelectron emission from real copper surfaces in sliding contact with polytetrafluoroethylene and polyimide. Tribol Int 47: 212-220 (2012)
[37] Wang Y X, Yan F Y. Tribological properties of transfer films of PTFE-based composites. Wear 261(11-12): 1359-1366 (2006)

[38] Şahin Y. Analysis of abrasive wear behavior of PTFE composite using Taguchi's technique. Cogent Eng 2(1): 1000510 (2015)

[39] Amrishraj D, Senthilvelan T. Development of wear mechanism maps for acrylonitrile butadiene styrene hybrid composites reinforced with nano zirconia and PTFE under dry sliding condition. $J$ Tribol 141(2): 021602 (2019)

[40] Unal H, Yetgin S H, Mimaroglu A, Sumer M. The effect of test parameters on friction and wear performance of PTFE and PTFE composites. J Reinforced Plast Compos 29(13): 1978-1986 (2010)

[41] Mazza L, Trivella A, Grassi R, Malucelli G. A comparison of the relative friction and wear responses of PTFE and a PTFE-based composite when tested using three different types of sliding wear machines. Tribol Int 90: 15-21 (2015)

[42] Chen B B, Wang J Z, Yan F Y. Microstructure of PTFE-based polymer blends and their tribological behaviors under aqueous environment. Tribol Lett 45(3): 387-395 (2012)

[43] Conte M, Pinedo B, Igartua A. Role of crystallinity on wear behavior of PTFE composites. Wear 307(1-2): 81-86 (2013)

[44] Lai S Q, Yue L, Li T S. Mechanism of filler action in reducing the wear of PTFE polymer by differential scanning calorimetry. J Appl Polym Sci 106(5): 30913097 (2007)

[45] Song Y L, Chen J, Li J L, Li H, Zhang J X. Dynamic thermal mechanical analysis and its application in the research on fluorinated polymer materials. Chem Prod Technol 23(1): 14-16, 60 (2016)

[46] Zhang G D, Fan L, Bai L, He M H, Zhai L, Mo S. Mesoscopic simulation assistant design of immiscible polyimide/BN blend films with enhanced thermal conductivity. Chin J Polym Sci 36(12): 1394-1402 (2018)

[47] Yoshizawa H, Chen Y L, Israelachvili J. Fundamental mechanisms of interfacial friction. 1. Relation between adhesion and friction. $J$ Phys Chem 97(16): 4128-4140 (1993)

[48] Richard S, Selwin Rajadurai J, Manikandan V. Effects of particle loading and particle size on tribological properties of biochar particulate reinforced polymer composites. J Tribol 139(1): 012202 (2017)

[49] Sahin Y. Analysis of abrasive wear of ptfee composites. Engineering 2: 1-15(2015).

[50] Kato K. Classification of wear mechanisms/models. Proc Inst Mech Eng J Eng Tribol 216(6): 349-355 (2002)

[51] Dong C L, Yuan C Q, Bai X Q, Qin H L, Yan X P. 
Investigating relationship between deformation behaviours and stick-slip phenomena of polymer material. Wear 376-377: 1333-1338 (2017)

[52] Samyn P, Schoukens G. Experimental extrapolation model for friction and wear of polymers on different testing scales. Int J Mech Sci 50(9): 1390-1403 (2008)

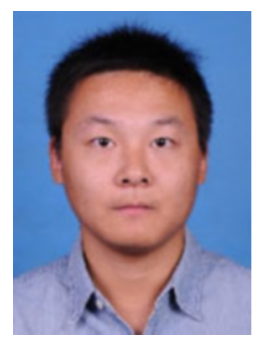

Chaojie XIE. He received his master degree in mechanical engineering from Beijing University of Chemical

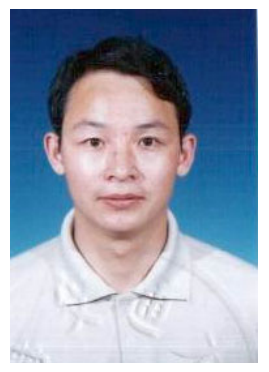

Kejian WANG. He received his master degree in mechanical engineering from BUCT in 1998 and the Ph.D. degree in material engineering from Shanghai Jiao Tong University, 2001, China. After working (20012002) as a lecturer in South China University of Technology, he moved

to Beijing Institute of Aeronautical Materials as a
[53] Liu Y, Xu J Q, Mutoh Y. Evaluation of fretting wear based on the frictional work and cyclic saturation concepts. Int J Mech Sci 50(5): 897-904 (2008)

[54] Feng C, Zhang D K, Chen K, Guo Y B. Study on viscoelastic friction and wear between friction linings and wire rope. Int J Mech Sci 142-143: 140-152 (2018)

Technology (BUCT) in 2020. He graduated from Changjiang University in mechanical engineering, 2018.

postdoctor and a senior engineer from January 2002 to May 2004. From 2004 to 2005, he worked as a Japan Society for the Promotion of Science (JSPS) postdoctor fellow in Japan Institute of Aeronautical and Space. After that, he acts as a research fellow, doctorate supervisor, and director of the Molding Manufacturing Research Center in BUCT up to now. His main research areas are polymeric composites and rheology, tribology, processing technology, and product design. 